\title{
Nonlinearly Self-Adjoint, Conservation Laws and Solutions for a Forced BBM Equation
}

\author{
Maria Luz Gandarias ${ }^{1}$ and Chaudry Masood Khalique ${ }^{2}$ \\ ${ }^{1}$ Departamento de Matemáticas, Universidad de Cádiz, P.O. Box 40, Puerto Real, 11510 Cádiz, Spain \\ ${ }^{2}$ International Institute for Symmetry Analysis and Mathematical Modelling, Department of Mathematical Sciences, \\ North-West University, Mafikeng Campus, Private Bag X 2046, Mmabatho 2735, South Africa
}

Correspondence should be addressed to Chaudry Masood Khalique; masood.khalique@nwu.ac.za

Received 31 January 2014; Accepted 2 March 2014; Published 3 April 2014

Academic Editor: Mariano Torrisi

Copyright (C) 2014 M. L. Gandarias and C. M. Khalique. This is an open access article distributed under the Creative Commons Attribution License, which permits unrestricted use, distribution, and reproduction in any medium, provided the original work is properly cited.

We study a forced Benjamin-Bona-Mahony (BBM) equation. We prove that the equation is not weak self-adjoint; however, it is nonlinearly self-adjoint. By using a general theorem on conservation laws due to Nail Ibragimov and the symmetry generators, we find conservation laws for these partial differential equations without classical Lagrangians. We also present some exact solutions for a special case of the equation.

\section{Introduction}

In a recent paper [1], Eloe and Usman have considered the damped externally excited Benjamin-Bona-Mahony (BBM) type equation given by

$$
u_{t}+u_{x}+2 b u u_{x}-c u_{x x}-d u-a u_{x x t}=\eta \cos k(x+\lambda t),
$$

where $c$ and $d$ are nonnegative constants that are proportional to the strength of the damping effect. Equation (1) was introduced to model long waves in nonlinear dispersive systems. Some special cases of (1) are studied in $[2,3]$. If $b=1 / 2$ and $a=1, c=0, d=0$, and $\eta=0$, then (1) reduces to the celebrated Benjamin-Bona-Mahony (BBM) equation

$$
u_{t}+u_{x}+u u_{x}-u_{x x t}=0 .
$$

The well-known BBM equation (2) was derived in [4] for moderately long wave equations in nonlinear dispersive systems. The authors derived three conservation laws for (2) and also considered the forcing equation. In [5], it was proved that these conservation laws are the only conservation laws admitted by the BBM equation. In [6], a family of $\mathrm{BBM}$ equations with strong nonlinear dispersive term was considered from the point of view of symmetry analysis. The symmetry reductions were derived from the optimal system of subalgebras and lead to systems of ordinary differential equations. For special values of the parameters of this equation, many exact solutions are expressed by various single and combined nondegenerative Jacobi elliptic function solutions and their degenerative solutions (soliton, kink, and compactons). In [7], nonlocal symmetries of a family of Benjamin-Bona-Mahony-Burgers equations were studied. In [8] for a family of Benjamin-Bona-Mahony equations with strong nonlinear dispersion, the subclass of equations which are self-adjoint was determined and some nontrivial conservation laws were derived. In [9], da Silva and Freire showed that the BBM equation is strictly self-adjoint and a conservation law obtained from the scaling invariance was established.

In [1], the authors have obtained an analytic steady state solution of (1) and they have studied properties of some travelling wave solutions using a perturbation method.

In [10], the first author of this paper introduced the definition of weak self-adjointness and showed that the substitution $v=h(u)$ can be replaced with a more general substitution, where $h$ involves not only the variable $u$ but also the independent variables $h=h(x, t, u)$. In [11], Ibragimov 
pointed out that, in constructing conservation laws, it is only important that $v$ does not vanish identically and introduced the definition of nonlinearly self-adjoint equation; that is, the substitution $v=h(u)$ can be replaced with a more general substitution, where $h$ involves not only the variable $u$ but also its derivatives as well as the independent variables; that is, $v=h\left(x, t, u, u_{t}, u_{x}, \ldots\right)$.

In this paper, we consider a generalization of the damped externally excited Benjamin-Bona-Mahony type equation (1), that is, the forced BMM type equation

$$
u_{t}+u_{x}+2 b u u_{x}-c u_{x x}-d u-a u_{x x t}=f(x, t),
$$

where $c$ and $d$ are nonnegative constants that are proportional to the strength of the damping effect and $f(x, t)$ is an arbitrary function of the variables $x$ and $t$.

The aim of this paper is to prove that (3) is nonlinearly self-adjoint. We determine, by using the Lie generators of (3) and the notation and techniques of [12], some nontrivial conservation laws for (3). Finally, we present some exact solutions for a special case of (3).

\section{Self-Adjoint and Nonlinearly Self-Adjoint Equations}

Consider an sth-order partial differential equation

$$
F\left(x, u, u_{(1)}, \ldots, u_{(s)}\right)=0
$$

with independent variables $x=\left(x^{1}, \ldots, x^{n}\right)$ and a dependent variable $u$, where $u_{(1)}=\left\{u_{i}\right\}, u_{(2)}=\left\{u_{i j}\right\}, \ldots$, denote the sets of the partial derivatives of the first, second, and so forth orders, $u_{i}=\partial u / \partial x^{i}, u_{i j}=\partial^{2} u / \partial x^{i} \partial x^{j}$. The adjoint equation to $(4)$ is

$$
F^{*}\left(x, u, v, u_{(1)}, v_{(1)}, \ldots, u_{(s)}, v_{(s)}\right)=0
$$

with

$$
F^{*}\left(x, u, v, u_{(1)}, v_{(1)}, \ldots, u_{(s)}, v_{(s)}\right)=\frac{\delta(v F)}{\delta u},
$$

where

$$
\frac{\delta}{\delta u}=\frac{\partial}{\partial u}+\sum_{s=1}^{\infty}(-1)^{s} D_{i_{1}} \cdots D_{i_{s}} \frac{\partial}{\partial u_{i_{1} \cdots i_{s}}}
$$

denotes the variational derivatives (the Euler-Lagrange operator) and $v$ is a new dependent variable. Here,

$$
D_{i}=\frac{\partial}{\partial x^{i}}+u_{i} \frac{\partial}{\partial u}+u_{i j} \frac{\partial}{\partial u_{j}}+\cdots
$$

are the total differentiations.

Definition 1. Equation (4) is said to be self-adjoint if the equation obtained from the adjoint equation (5) by the substitution $v=u$,

$$
F^{*}\left(x, u, u, u_{(1)}, u_{(1)}, \ldots, u_{(s)}, u_{(s)}\right)=0
$$

is identical to the original equation (4).
Definition 2. Equation (4) is said to be weak self-adjoint if the equation obtained from the adjoint equation (5) by the substitution $v=h(x, t, u)$, with a certain function $h(x, t, u)$ such that $h_{x}(x, t, u) \neq 0,\left(\right.$ or $\left.h_{t}(x, t, u) \neq 0\right)$ and $h_{u}(x, t, u) \neq 0$, is identical to the original equation.

Definition 3. Equation (4) is said to be nonlinearly selfadjoint if the equation obtained from the adjoint equation (5) by the substitution $v=h\left(x, t, u, u_{(1)}, \ldots\right)$, with a certain function $h\left(x, t, u, u_{(1)}, \ldots\right)$ such that $h\left(x, t, u, u_{(1)}, \ldots\right) \neq 0$, is identical to the original equation (4).

2.1. The Subclass of Nonlinearly Self-Adjoint Equations. Let us single out some nonlinearly self-adjoint equations from the equations of the form (3). Equation (6) yields

$$
\begin{aligned}
F^{*}= & \frac{\delta}{\delta u}\left[v \left(u_{t}+u_{x}+2 b u u_{x}-c u_{x x}\right.\right. \\
& \left.\left.-d u-a u_{x x t}-f(x, t)\right)\right] \\
= & -c v_{x x}-2 b u v_{x}-v_{x}+a v_{t x x}-v_{t}-d v
\end{aligned}
$$

Setting $v=h(x, t, u)$ in $(10)$, we get

$$
\begin{aligned}
& a h_{u u} u_{t} u_{x x}-c h_{u} u_{x x}+a h_{t u} u_{x x}+a h_{u u u} u_{t}\left(u_{x}\right)^{2} \\
& \quad-c h_{u u}\left(u_{x}\right)^{2}+a h_{t u u}\left(u_{x}\right)^{2}+2 a h_{u u} u_{t x} u_{x} \\
& \quad+2 a h_{u u x} u_{t} u_{x}-2 b h_{u} u u_{x}-2 c h_{u x} u_{x}-h_{u} u_{x} \\
& \quad+2 a h_{t u x} u_{x}+a h_{u} u_{t x x}+2 a h_{u x} u_{t x}+a h_{u x x} u_{t} \\
& \quad-h_{u} u_{t}-2 b h_{x} u-c h_{x x}-h_{x}+a h_{t x x}-h_{t}-d h=0 .
\end{aligned}
$$

Now, we assume that

$$
F^{*}-\lambda\left(u_{t}+u_{x}+2 b u u_{x}-c u_{x x}-d u-a u_{x x t}-f(x, t)\right)=0 \text {, }
$$

where $\lambda$ is an undetermined coefficient. Condition (12) reads

$$
\begin{aligned}
& c u_{x x} \lambda-2 b u u_{x} \lambda-u_{x} \lambda+a u_{t x x} \lambda-u_{t} \lambda \\
& +d u \lambda+f \lambda+a h_{u u} u_{t} u_{x x}-c h_{u} u_{x x} \\
& +a h_{t u} u_{x x}+a h_{u u u} u_{t}\left(u_{x}\right)^{2}-c h_{u u}\left(u_{x}\right)^{2} \\
& \quad+a h_{t u u}\left(u_{x}\right)^{2}+2 a h_{u u} u_{t x} u_{x}+2 a h_{u u x} u_{t} u_{x} \\
& \quad-2 b h_{u} u u_{x}-2 c h_{u x} u_{x}-h_{u} u_{x}+2 a h_{t u x} u_{x} \\
& +a h_{u} u_{t x x}+2 a h_{u x} u_{t x}+a h_{u x x} u_{t} \\
& \quad-h_{u} u_{t}-2 b h_{x} u-c h_{x x}-h_{x}+a h_{t x x}-h_{t}-d h=0
\end{aligned}
$$

Comparing the coefficients for the different derivatives of $u$, we obtain

$$
\begin{gathered}
\lambda=-h_{u}, \\
h=c_{2} e^{2 c t / a} u+\beta,
\end{gathered}
$$


where $f=f(x, t)$ and $\beta(x, t)$ satisfy the following conditions:

$$
\begin{gathered}
-2 c_{2} d e^{2 c t / a}-\frac{2 c c_{2} e^{2 c t / a}}{a}-2 b \beta_{x}=0, \\
-c_{2} f e^{2 c t / a}-\beta d-\beta_{x x} c-\beta_{x}+a \beta_{t x x}-\beta_{t}=0 .
\end{gathered}
$$

From above, we get that

$$
\beta=f_{3}-\frac{\left(a c_{2} d+c c_{2}\right) e^{2 c t / a} x}{a b}
$$

with $f_{3}=f_{3}(t)$ and the following condition must be satisfied:

$$
\begin{aligned}
& \frac{c_{2} d^{2} e^{2 c t / a} x}{b}+\frac{3 c c_{2} d e^{2 c t / a} x}{a b}+\frac{2 c^{2} c_{2} e^{2 c t / a} x}{a^{2} b} \\
& -c_{2} f e^{2 c t / a}+\frac{c_{2} d e^{2 c t / a}}{b}+\frac{c c_{2} e^{2 c t / a}}{a b}-f_{3 t}-d f_{3}=0 .
\end{aligned}
$$

We can now state the following theorem.

Theorem 4. Equation (3) is nonlinearly self-adjoint with

$$
h=-\frac{\left(a c_{2} d+c c_{2}\right) e^{2 c t / a} x}{a b}+c_{2} e^{2 c t / a} u+f_{3}
$$

for any functions $f=f(x, t)$ and $f_{3}(t)$ satisfying condition (17).

In particular, we can state the following theorem.

Theorem 5. Equation (3) is nonlinearly self-adjoint for any arbitrary function $f=f(x, t)$ with

$$
h=c_{3} e^{-d t} \text {. }
$$

\section{Conservation Laws: General Theorem}

We use the following theorem on conservation laws proved in [12].

Theorem 6. Any Lie point, Lie-Bäcklund, or non-local symmetry

$$
X=\xi^{i}\left(x, u, u_{(1)}, \ldots\right) \frac{\partial}{\partial x^{i}}+\eta\left(x, u, u_{(1)}, \ldots\right) \frac{\partial}{\partial u}
$$

of (4) provides a conservation law $D_{i}\left(C^{i}\right)=0$ for system (4), (5). The conserved vector is given by

$$
\begin{aligned}
C^{i}= & \xi^{i} \mathscr{L}+W\left[\frac{\partial \mathscr{L}}{\partial u_{i}}-D_{j}\left(\frac{\partial \mathscr{L}}{\partial u_{i j}}\right)+D_{j} D_{k}\left(\frac{\partial \mathscr{L}}{\partial u_{i j k}}\right)-\cdots\right] \\
& +D_{j}(W)\left[\frac{\partial \mathscr{L}}{\partial u_{i j}}-D_{k}\left(\frac{\partial \mathscr{L}}{\partial u_{i j k}}\right)+\cdots\right] \\
& +D_{j} D_{k}(W)\left[\frac{\partial \mathscr{L}}{\partial u_{i j k}}-\cdots\right]+\cdots
\end{aligned}
$$

where $W$ and $\mathscr{L}$ are defined as follows:

$$
W=\eta-\xi^{j} u_{j}, \quad \mathscr{L}=v F\left(x, u, u_{(1)}, \ldots, u_{(s)}\right) .
$$

Let us apply Theorem 6 to the nonlinearly self-adjoint equation:

$$
u_{t}+u_{x}+2 b u u_{x}-c u_{x x}-d u-a u_{x x t}=f(x, t),
$$

where

$$
\mathscr{L}=\left(u_{t}+u_{x}+2 b u u_{x}-c u_{x x}-d u-a u_{x x t}-f(x, t)\right) v,
$$

provided by the generator

$$
\mathbf{v}=k_{1} \frac{\partial}{\partial t}+k_{2} \frac{\partial}{\partial x} .
$$

Here, $f=f(x, t)$ must satisfy $k_{1} f_{t}+k_{2} f_{x}=0$. We get the conservation law

$$
D_{t}\left(C^{1}\right)+D_{x}\left(C^{2}\right)=0
$$

with

$$
\begin{gathered}
C^{1}=-k k_{1} e^{-d t}(d u+f)+D_{x}(B), \\
C^{2}=k e^{-d t}\left(c d k_{1} u_{x}+a d k_{1} u_{t x}-b d k_{1} u^{2}\right. \\
\left.-d k_{1} u-f k_{2}\right)-D_{t}(B),
\end{gathered}
$$

where

$$
\begin{gathered}
B=\left(k e ^ { - d t } \left(a k_{2} u_{x x}-3 c k_{1} u_{x}-2 a k_{1} u_{t x}+3 b k_{1} u^{2}\right.\right. \\
\left.\left.-3 k_{2} u+3 k_{1} u\right)\right)(3)^{-1} .
\end{gathered}
$$

We simplify the conserved vector by transferring the terms of the form $D_{x}(\cdots)$ from $C^{1}$ to $C^{2}$ and obtain

$$
\begin{gathered}
C^{1}=-k k_{1} e^{-d t}(d u+f) \\
C^{2}=k e^{-d t}\left(c d k_{1} u_{x}+a d k_{1} u_{t x}\right. \\
\left.-b d k_{1} u^{2}-d k_{1} u-f k_{2}\right) .
\end{gathered}
$$

\section{Exact Solutions}

In this section, we obtain exact solutions of (3) when $d=0$ and $f=0$; that is, we consider the following equation:

$$
u_{t}+u_{x}+2 b u u_{x}-c u_{x x}-a u_{x x t}=0 .
$$

This equation has two translation symmetries; namely, $X_{1}=$ $\partial / \partial x$ and $X_{2}=\partial / \partial t$. We first use these two symmetries and transform (30) into an ordinary differential equation. Then, employing the simplest equation method, we obtain exact solutions.

4.1. Symmetry Reduction of (30). The symmetry $v X_{1}+X_{2}$ gives rise to the group-invariant solution

$$
u=F(z),
$$

where $z=x-v t$ is an invariant of $v X_{1}+X_{2}$. Substitution of (31) into (30) results in the nonlinear third-order ordinary differential equation

$$
a v F^{\prime \prime \prime}(z)-c F^{\prime \prime}(z)+2 b F(z) F^{\prime}(z)+(1-v) F^{\prime}(z)=0 .
$$


4.2. Exact Solutions Using Simplest Equation Method. Let us briefly recall the simplest equation method $[13,14]$ here. Consider the solutions of (32) in the form

$$
F(z)=\sum_{i=0}^{M} A_{i}(H(z))^{i}
$$

where $H(z)$ satisfies a Bernoulli or Riccati equation, $M$ is a positive integer that can be determined by a balancing procedure [14], and the coefficients $A_{0}, \ldots, A_{M}$ are parameters to be determined.

The Bernoulli equation we consider here is given by

$$
H^{\prime}(z)=\alpha H(z)+\beta H^{2}(z),
$$

which has a solution in the form

$$
H(z)=\alpha\left\{\frac{\cosh [\alpha(z+C)]+\sinh [\alpha(z+C)]}{1-\beta \cosh [\alpha(z+C)]-\beta \sinh [\alpha(z+C)]}\right\} .
$$

For the Riccati equation

$$
H^{\prime}(z)=\alpha H^{2}(z)+\beta H(z)+\gamma
$$

we will use the two solutions

$$
\begin{gathered}
H(z)=-\frac{\beta}{2 \alpha}-\frac{\theta}{2 \alpha} \tanh \left[\frac{1}{2} \theta(z+C)\right], \\
H(z)=-\frac{\beta}{2 \alpha}-\frac{\theta}{2 \alpha} \tanh \left(\frac{1}{2} \theta z\right) \\
+\frac{\operatorname{sech}(\theta z / 2)}{C \cosh (\theta z / 2)-(2 \alpha / \theta) \sinh (\theta z / 2)},
\end{gathered}
$$

where $\theta^{2}=\beta^{2}-4 \alpha \gamma$ and $C$ is a constant of integration.

4.2.1. Solutions of (30) Using Bernoulli Equation as the Simplest Equation. In this case, the balancing procedure [14] gives $M=2$ and therefore the solutions of (32) are of the form

$$
F(z)=A_{0}+A_{1} H+A_{2} H^{2}
$$

Now, substituting (38) into (32) and making use of (34) and then equating all coefficients of the functions $H^{i}$ to zero, we obtain an algebraic system of equations in terms of $A_{0}, A_{1}$, and $A_{2}$.

Solving this system of algebraic equations, with the aid of Mathematica, we obtain

$$
\begin{gathered}
b=-\frac{6 a v \beta^{2}}{A_{2}}, \quad c=-5 a v \alpha, \\
A_{0}=\frac{A_{2}\left(-v+1+6 a v \alpha^{2}\right)}{12 a v \beta^{2}}, \quad A_{1}=\frac{2 A_{2} \alpha}{\beta} .
\end{gathered}
$$

Thus, a solution of (30) is

$$
\begin{aligned}
& u(t, x) \\
& =A_{0}+A_{1} \alpha\left\{\frac{\cosh [\alpha(z+C)]+\sinh [\alpha(z+C)]}{1-\beta \cosh [\alpha(z+C)]-\beta \sinh [\alpha(z+C)]}\right\} \\
& \quad+A_{2} \alpha^{2}\left\{\frac{\cosh [\alpha(z+C)]+\sinh [\alpha(z+C)]}{1-\beta \cosh [\alpha(z+C)]-\beta \sinh [\alpha(z+C)]}\right\}^{2},
\end{aligned}
$$

where $z=x-v t$ and $C$ is a constant of integration.

4.2.2. Solutions of (30) Using Riccati Equation as the Simplest Equation. The balancing procedure yields $M=2$ so the solutions of (32) are of the form

$$
F(z)=A_{0}+A_{1} H+A_{2} H^{2} .
$$

Again substituting (41) into (32) and making use of the Riccati equation (36), we obtain, as before, an algebraic system of equations in terms of $A_{0}, A_{1}, A_{2}$. Solving the algebraic system of equations, one obtains

$$
\begin{gathered}
b=-\frac{6 a v \alpha^{2}}{A_{2}}, \quad c=-\frac{5 a v\left(A_{1} \alpha-A_{2} \beta\right)}{A_{2}}, \\
\gamma=-\frac{A_{1}\left(A_{1} \alpha-2 A_{2} \beta\right)}{4 A_{2}^{2}},
\end{gathered}
$$

$$
\begin{aligned}
& A_{0} \\
& =-\frac{3 a v \alpha^{2} A_{1}^{2}-12 a v A_{1} \alpha \beta A_{2}+6 a v A_{2}^{2} \beta^{2}+\nu A_{2}^{2}-A_{2}^{2}}{12 a v A_{2} \alpha^{2}},
\end{aligned}
$$

and hence solutions of (30) are

$$
\begin{aligned}
u(t, x)= & A_{0}+A_{1}\left\{-\frac{\beta}{2 \alpha}-\frac{\theta}{2 \alpha} \tanh \left[\frac{1}{2} \theta(z+C)\right]\right\} \\
& +A_{2}\left\{-\frac{\beta}{2 \alpha}-\frac{\theta}{2 \alpha} \tanh \left[\frac{1}{2} \theta(z+C)\right]\right\}^{2}, \\
u(t, x)= & A_{0}+A_{1}\left\{-\frac{\beta}{2 \alpha}-\frac{\theta}{2 \alpha} \tanh \left(\frac{1}{2} \theta z\right)\right. \\
& +A_{2}\left\{-\frac{\beta}{C \alpha}-\frac{\theta}{2 \alpha} \tanh \left(\frac{1}{2} \theta z\right)\right. \\
& \left.+\frac{\operatorname{sech}(\theta z / 2)}{C \cosh (\theta z / 2)-(2 \alpha / \theta) \sinh (\theta z / 2)}\right\}^{2},
\end{aligned}
$$

where $z=x-v t$ and $C$ is a constant of integration.

\section{Conclusions}

We have proved that the generalized forced BBM equation (3) is nonlinearly self-adjoint. We have determined, by using 
the Lie generators of (3) and the notation and techniques of [12], some nontrivial conservation laws for (3). Finally, we presented some exact solutions for a special case of (3).

\section{Conflict of Interests}

The authors declare that there is no conflict of interests regarding the publication of this paper.

\section{Acknowledgments}

Maria Luz Gandarias acknowledges the support of Junta de Andalucía Group FQM-201 and Chaudry Masood Khalique thanks the North-West University, Mafikeng Campus, for its continued support.

\section{References}

[1] P. Eloe and M. Usman, "Bifurcations in steady state solutions of a class of nonlinear dispersive wave equations," Nonlinear Studies, vol. 19, no. 2, pp. 215-224, 2012.

[2] M. Usman and B. Zhang, "Forced oscillations of a class of nonlinear dispersive wave equations and their stability," Journal of Systems Science \& Complexity, vol. 20, no. 2, pp. 284-292, 2007.

[3] M. Usman and B.-Y. Zhang, "Forced oscillations of the Korteweg-de vries equation on a bounded domain and their stability," Discrete and Continuous Dynamical Systems A, vol. 26, no. 4, pp. 1509-1523, 2010.

[4] T. B. Benjamin, J. L. Bona, and J. J. Mahony, "Model equations for long waves in nonlinear dispersive systems," Philosophical Transactions of the Royal Society of London A: Mathematical and Physical Sciences, vol. 272, no. 1220, pp. 47-78, 1972.

[5] P. J. Olver, "Euler operators and conservation laws of the BBM equation," Mathematical Proceedings of the Cambridge Philosophical Society, vol. 85, no. 1, pp. 143-160, 1979.

[6] M. S. Bruzón, M. L. Gandarias, and J. C. Camacho, "Symmetry analysis and solutions for a generalization of a family of BBM equations," Journal of Nonlinear Mathematical Physics, vol. 15, supplement 3, pp. 81-90, 2008.

[7] M. S. Bruzón and M. L. Gandarias, "Nonlocal symmetries for a family Benjamin-Bona-Mahony-Burgers equations. Some exact solutions," International Journal of Applied Mathematics and Informatics, vol. 5, pp. 180-187, 2011.

[8] M. S. Bruzon and M. L. Gandarias, "On the group classification and conservation laws of the self-adjoint of a family BenjaminBona-Mahony equations," International Journal of Mathematical Models and Methods in Applied Sciences, vol. 4, pp. 527-534, 2012.

[9] P. L. da Silva and I. L. Freire, "Strict self-adjointness and shallow water models," http://arxiv.org/abs/1312.3992.

[10] M. L. Gandarias, "Weak self-adjoint differential equations," Journal of Physics A: Mathematical and Theoretical, vol. 44, no. 26, Article ID 262001, 2011.

[11] N. H. Ibragimov, "Nonlinear self-adjointness and conservation laws," Journal of Physics A: Mathematical and Theoretical, vol. 44, no. 43, Article ID 432002, 2011.

[12] N. H. Ibragimov, "A new conservation theorem," Journal of Mathematical Analysis and Applications, vol. 333, no. 1, pp. 311$328,2007$.
[13] N. A. Kudryashov, "Simplest equation method to look for exact solutions of nonlinear differential equations," Chaos, Solitons and Fractals, vol. 24, no. 5, pp. 1217-1231, 2005.

[14] N. K. Vitanov, "Application of simplest equations of Bernoulli and Riccati kind for obtaining exact traveling-wave solutions for a class of PDEs with polynomial nonlinearity," Communications in Nonlinear Science and Numerical Simulation, vol. 15, no. 8, pp. 2050-2060, 2010. 


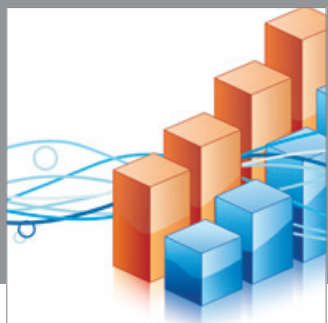

Advances in

Operations Research

mansans

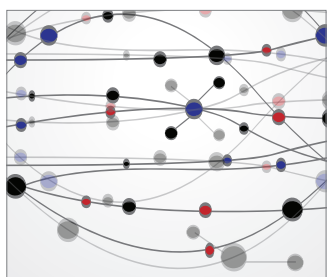

The Scientific World Journal
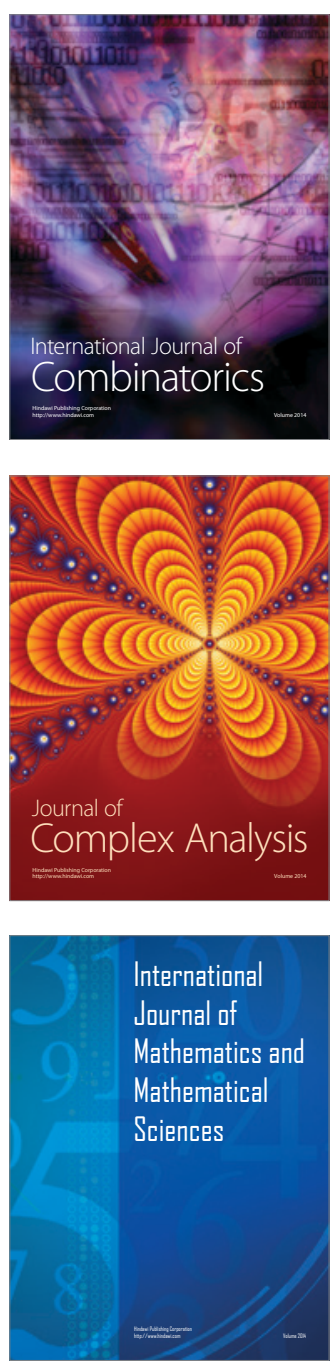
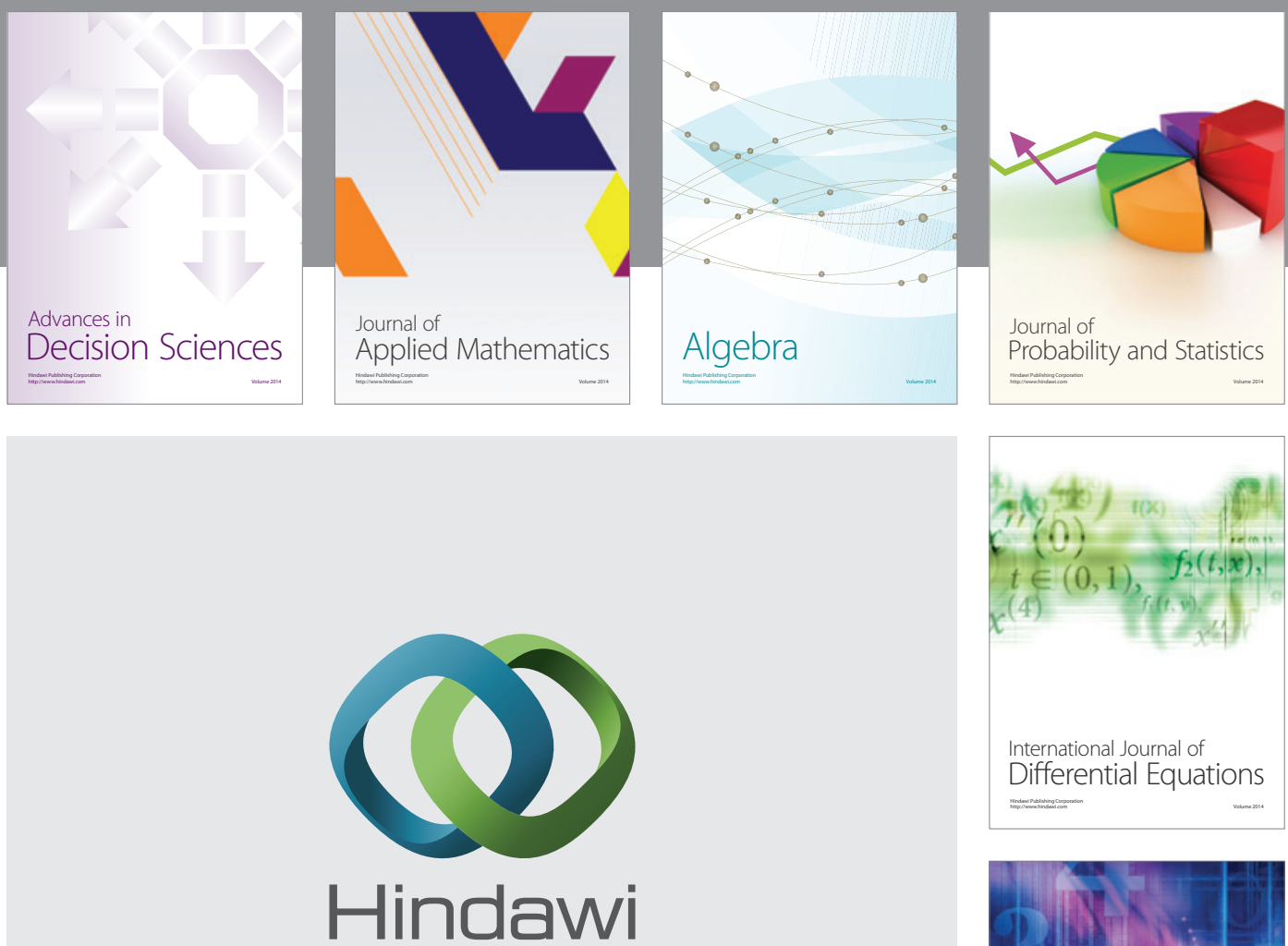

Submit your manuscripts at http://www.hindawi.com
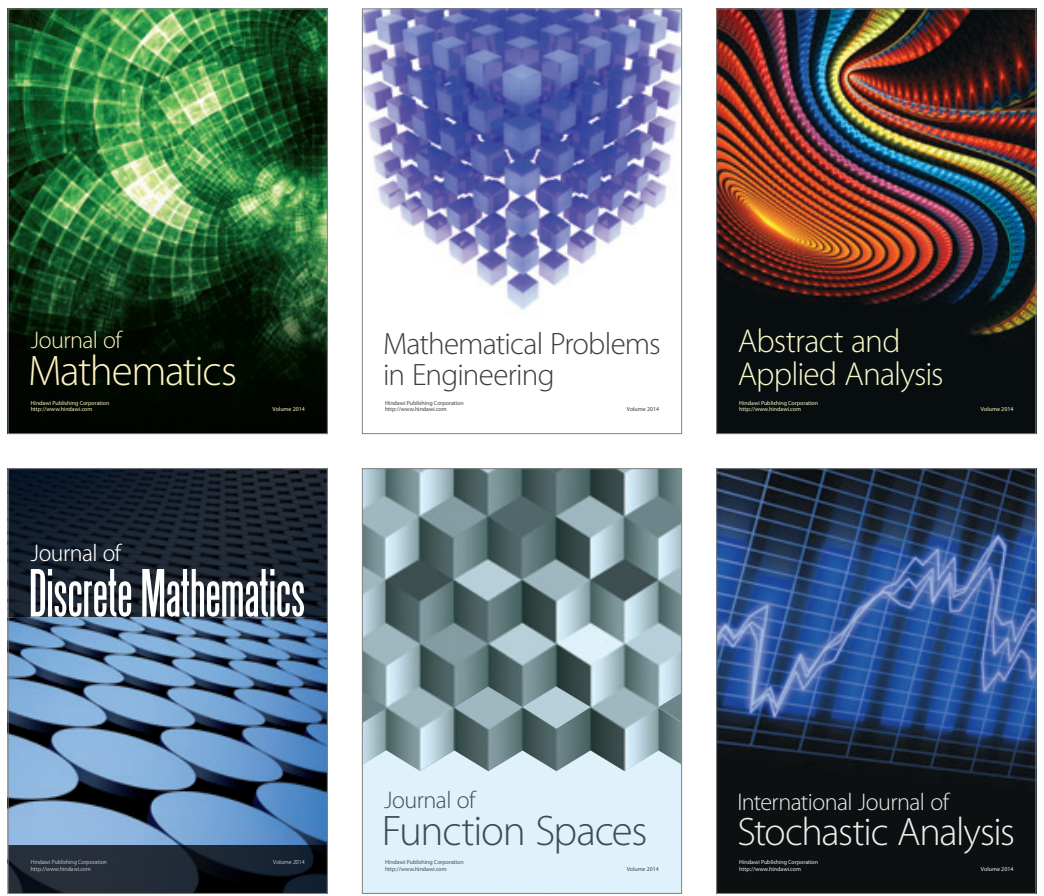

Journal of

Function Spaces

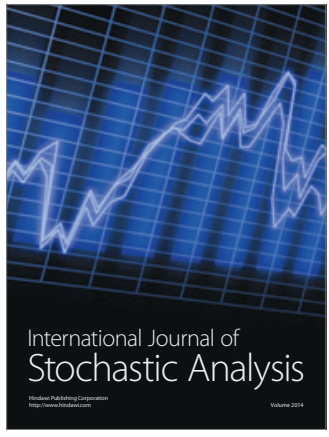

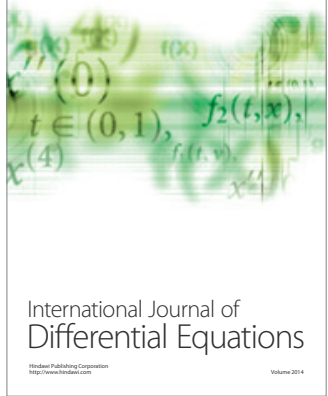
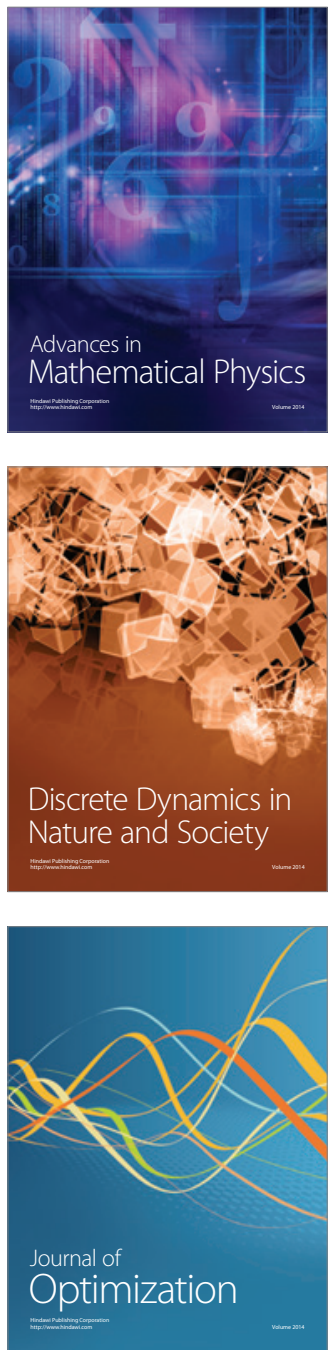